\title{
Adherence to the Mediterranean diet is associated with a lower risk of body-shape changes in Croatian patients treated with combination antiretroviral therapy
}

\author{
Drago Turčinov $\cdot$ Christine Stanley $\cdot$ \\ George W. Rutherford · Thomas E. Novotny • \\ Josip Begovac
}

Received: 10 September 2006/ Accepted: 6 March 2009/Published online: 26 March 2009

(C) The Author(s) 2009. This article is published with open access at Springerlink.com

\begin{abstract}
Lipoatrophy and lipohypertrophy have been observed during long-term combination antiretroviral therapy (CART). We investigated whether consumption of a Mediterranean diet is associated with lower risk of bodyshape changes in Croatian patients treated with CART. Between May 2004 and June 2005, we conducted a crosssectional study of 136 adults with HIV-1 infection who were treated with CART for at least 1 year. Lipoatrophy and lipohypertrophy were assessed by self-report and physical examination. Adherence to a Mediterranean diet was determined by a 150 -item questionnaire; a $0-9$ point diet scale was created that stratified respondents as having low adherence ( $<4$ points) and moderate to high adherence ( $\geq 4$ points). Lipoatrophy was present in $41 \%$ and lipohypertrophy in $32 \%$ of participants. Non-smokers with a dietary score $\geq 4$ had the lowest risk for lipoatrophy. Stavudine use, female gender, and duration of CART were also independently associated with a higher risk of lipoatrophy. A dietary score of $\geq 4$ was associated with lower risk of lipohypertrophy (adjusted OR $0.3,95 \%$ CI $0.1-0.7 ; P=0.012$ ). Female gender, longer duration of CART, and longer known duration of HIV infection prior to CART were also independently associated with higher risk of lipohypertrophy. In conclusion, Croatians who did not smoke and moderately or highly adhered to the Mediterranean diet were least likely to have the clinical
\end{abstract}

D. Turčinov $\cdot$ J. Begovac $(\bowtie)$

University Hospital of Infectious Diseases, Mirogojska 8,

Zagreb 10000, Croatia

e-mail: josip.begovac@gmail.com

C. Stanley · G. W. Rutherford - T. E. Novotny

San Francisco School of Medicine, University of California,

San Francisco, CA, USA syndrome of lipoatrophy. Moderate to high adherence to a Mediterranean diet was associated with a lower risk of lipohypertrophy.

Keywords HIV · Antiretrovirals · Lipoatrophy · Lipohypertrophy $\cdot$ Mediterranean diet
Abbreviations
CART Combination antiretroviral therapy
HIV Human immunodeficiency virus
BMI Body mass index
MET Metabolic equivalence
AIDS Acquired immunodeficiency syndrome
UHID University hospital for Infectious Diseases
IQR Inter-quartile ranges
DEXA Dual energy X-ray absorptiometry
NNRTI Non-nucleoside reverse transcriptase inhibitors
PI Protease inhibitor

\section{Introduction}

HIV-associated lipodystrophy, also known as fat redistribution syndrome, is an important complication of combination antiretroviral therapy (CART). It is characterized by sustained changes in body fat distribution (lipoatrophy and lipohypertrophy), and is often accompanied by dyslipidemia and insulin resistance [1-4]. Peripheral lipoatrophy is characterized by wasting in the face, arms, buttocks and legs, and lipohypertrophy by increased deposition of fat in the abdomen, breasts, and dorso-cervical spine and by the appearance of lipomas [1, $5,6]$. Recent studies have shown that there is no reciprocal association between peripheral lipoatrophy and central lipohypertrophy [4, 7-9]. Body fat changes were associated with different host factors, severity of HIV disease, and 
type of treatment. Only a few studies have investigated the relationship of dietary intake with body fat changes and metabolic abnormalities in HIV-infected patients [10-14].

The traditional Mediterranean diet refers to dietary patterns observed in olive-growing areas of the Mediterranean region [15]. These dietary patterns include a high intake of vegetables, legumes, fresh fruits and nuts, cereals, and olive oil; a moderate intake of fish; a low-tomoderate intake of dairy products and wine; and a low intake of meat and poultry [16]. Most research on the Mediterranean diet has focused on mortality [16-18], prevention of coronary heart disease [19-21], and cancer prevention [22, 23].

We conducted a cross-sectional study of the relationship between adherence to a Mediterranean diet and body-shape changes in HIV infected patients treated with CART in Croatia.

\section{Methods}

\section{Setting}

Croatia is a Southeastern European upper-middle-income country with a population of just over 4.4 million. It can be divided into three geographic regions: Mediterranean coastal in the south, Pannonian continental in the north, and mountainous in the center. The Croatian diet can vary by region with persons living along the Adriatic Coast eating a diet more closely resembling the classic Mediterranean diet and those living in the continental and mountain regions eating a more classically Western diet.

Between 1985 and 2005 a total of 553 patients were diagnosed with HIV infection in Croatia. Of those 239 had an AIDS-defining illness and 127 died.

\section{Subjects}

Patients with documented HIV-1 infection were recruited from the Outpatient HIV/AIDS Department at the University Hospital of Infectious Diseases (UHID) in Zagreb, Croatia, from May 2004 to June 2005. Because all HIVinfected patients in Croatia are treated at UHID, we were able to enroll subjects from throughout the country. Included in the study were patients $\geq 18$ years old, who had taken CART for at least 1 year. Pregnant and breast feeding women, patients with uncontrolled opportunistic infections, and patients with disseminated malignancies were excluded. We had 169 patients who met the eligibility criteria. The study protocol was approved by the institutional review boards of UHID and the University of California, San Francisco.
Clinical, laboratory and lifestyle data

We administered a standard questionnaire to collect data on age, gender, education, family history of obesity, smoking, mode of infection, diet, current antiretroviral regimen and other treatments during a routine outpatient follow-up visit at UHID. A physical examination was performed that included measurements of waist and hip circumference (in $\mathrm{cm}$ ), weight (in $\mathrm{kg}$ ) and height (in $\mathrm{cm}$ ). Blood was drawn for determination of current CD4 cell count and HIV-1 RNA levels. CD4 cell counts were determined by flow cytometry and HIV-1 RNA levels were determined by polymerase chain reaction (Roche Amplicor Monitor) with the lower limit of detection of 50 copies/ml. Data on the known duration of HIV infection, history of AIDS-defining illness, weight and height prior to CART, peak HIV RNA level, lowest ever CD4 cell count, and past history of antiretroviral treatment were collected by reviewing patients' medical records. Treatment with a particular antiretroviral drug or drug class was considered present if the drug was administered for more than 6 months.

We assessed physical activity using the short version of the International Physical Activity Questionnaire [24]. Energy expenditure was calculated by weighting each type of activity by its energy requirements defined in metabolic equivalents (MET) [25].

Definition and assessment of body-shape changes measurements

We defined body-shape changes based on self-report confirmed by subsequent clinical examination by a physician. Body areas were evaluated using criteria developed for the US-based HIV Outpatient Study [26]. To reduce the influence of mild physical changes, we also used the same grading system [26]. Briefly, each participant was asked to report whether he had any changes in fat since starting CART in the face, legs, arms, buttock (atrophy), neck, chest, abdomen, or presence of lipomas (hypertrophy). Each sign of fat accumulation or fat wasting was rated as absent, mild, moderate or severe by the participant and confirmed by the physician. Participants reporting no changes or only mild changes in different body areas were categorized as having "no sign." Participants were classified in the "lipoatrophy" or "lipohypertrophy" group if they had at least one severe sign or one moderate sign in one area plus at least one subtle or moderate sign in another body area. Participants with mixed patterns were included in both groups.

Dietary assessment

Dietary intake was assessed through a previously validated 150 item food frequency questionnaire provided by 
Antonia Trichopoulou. The questionnaire was translated into Croatian and one of the authors (DT) interviewed patients. Nutrient intakes were calculated using food composition tables specific to Croatia. For each of the items in the questionnaire, subjects reported frequency of consumption and portion size, and the average monthly intake was divided into daily portions. To assist in accurate determination of portions, we provided 76 photographs depicting typical portion sizes. Items were divided into 12 food groups: potatoes, vegetables, legumes, fruit and nuts, dairy products, cereals, meat, poultry, fish, olive oil, eggs and alcoholic beverages. For each participant, intake of each of the indicated groups in grams per day and total energy intake were calculated. Potatoes were added to the cereal group and poultry was combined with meat to form one category. A ratio of monounsaturated fats to saturated fats was also calculated. We used a 10-point Mediterranean diet scale developed by Trichopoulou et al. [16]. For each subject, a value of 0 or 1 was determined for each of the nine components of the Mediterranean diet. The gender specific median consumption value was used as the cutoff point in each food category. For the six 'beneficial' categories (vegetable, legumes, fruits and nuts, cereal, fish and monounsaturated fat to saturated fat ratio) a value of 0 was given to subjects who consumed an amount below the median. For the two 'detrimental' categories (meat which included poultry, and dairy), a value of 1 was assigned to subjects who consumed an amount below the median for each of these categories. For ethanol consumption, a value of 1 was given to men who consumed 10-50 g/day and to women who consumed 5-25 g/day. The Mediterranean diet score ranged from 0 to 9 , with higher scores indicating greater adherence to the traditional Mediterranean diet. Because of the small number of participants in our study, we dichotomized the Mediterranean diet score into below the median $(<4$ points, indicating low adherence to the Mediterranean diet) and at or above the median ( $\geq 4$ points, indicating moderate to high adherence to the Mediterranean diet).

\section{Statistical analysis}

We used frequency distributions for descriptive analysis and medians and inter-quartile ranges (IQR) for continuous variables such as dietary and energy expenditure. To compare different characteristics of participants with lipoatrophy or lipohypertrophy to those without these conditions we used the Wilcoxon rank sum test for continuous variables and Fisher's exact test or the Chi-square test for categorical variables. In a multivariable logistic regression analysis we evaluated factors associated with lipoatrophy and lipohypertrophy separately. The dichotomized Mediterranean diet score was our main independent variable of interest. We screened for potential confounding variables by bivariate analysis and included those with a $P$-value $<0.25$ in the initial models. We applied a stepwise procedure that also included interaction terms and then used backward elimination of terms according to significance of the likelihood ratio test. There was evidence of nonlinearity for the duration of CART in the lipohypertrophy regression model, hence we dichotomized this variable into $<25$ months and $\geq 25$ months of treatment. Variables with a $P$-value $<0.05$ were retained in the final model. The interaction between stavudine and non-nucleoside reverse transcriptase inhibitors (NNRTI) use was significant $(P=0.05)$, suggesting that NNRTIs were associated with lipoatrophy only when stavudine was used. Because of this, we did not include the use of NNRTIs in our final lipoatrophy multivariate model. There was a significant interaction between smoking and the Mediterranean diet score in the lipoatrophy model. We assessed the adequacy of the final models by the HosmerLemeshow test and checked for outliers and multi-colinearity. All analyses were performed using the SAS statistical package (SAS System for Windows, Release 8.02, SAS Institute, Cary, NC).

\section{Results}

\section{Study participants}

Of 169 eligible patients, 136 (80\%) agreed to interview, examination, and review of their medical and database records. All participants were citizens of Croatia with a median age of 43 (IQR 36-50) years, and 108 (79\%) were males. Forty-three percent acquired HIV through heterosexual sex, $41 \%$ through sex between men and $10 \%$ through injecting drugs.

Sixty-five (48\%) of the participants were from the Adriatic coastal regions of Croatia, while 71 (52\%) were from the continental and mountainous areas of the country. The median Mediterranean diet score was 4 (IQR, 3-5.5) and $92(68 \%)$ participants had a Mediterranean diet score $\geq 4$. The median lowest ever CD4 cell count was 57.5 cells/ $\mathrm{mm}^{3}$ (IQR, 16-145) and the median current CD4 cell count was 356.5 cells $/ \mathrm{mm}^{3}$ (IQR, 244.5-486). There was no significant difference in the lowest ever and current CD4 cell count in participants with and without lipoatrophy or lipohypertrophy. The median peak plasma viral load was $5.6 \log _{10} / \mathrm{ml}$ (IQR, 5.1-5.9), and there was no significant difference in peak viral load between participants with and without lipoatrophy and lipohypertrophy.

The median duration of CART was 47 months (IQR, 23-67). There were 45 (33\%) participants who used only protease inhibitor (PI) based CART regiments, $34(25 \%)$ who used only NNRTIs regiments and 57 (42\%) who used 
both. Among PI-experienced participants 35\% used indinavir, 27\% lopinavir/ritonavir and 26\% indinavir/ritonavir. More participants had a history of efavirenz $(67,49 \%)$ use than of nevirapine $(27,20 \%)$. Of the 131 participants taking CART at the time of the interview, $43 \%$ were on treatment with a PI, 53\% were taking NNRTI-based therapy, and $4 \%$ were using both an NNRTI plus a PI. The most common current CART regimen was zidovudine plus lamivudine plus efavirenz (23\%), followed by zidovudine plus lamivudine plus lopinavir/ritonavir (16\%), zidovudine plus lamivudine plus nevirapine $(13 \%)$ and abacavir plus lamivudine plus efavirenz $(11 \%)$.

\section{Body-shape changes}

According to our study definitions (Table 1), 56 (41\%) participants had lipoatrophy and 44 (32\%) had lipohypertrophy. Severe signs of lipoatrophy were found most frequently in the face $(n=14)$, followed by thighs ( $n=13)$ and buttocks $(n=12)$. Severe signs of lipohyperthrophy were found most frequently in the abdomen $(n=21)$. Thirty-one participants $(23 \%)$ had only signs of peripheral fat atrophy, $19(14 \%)$ had only signs of lipohypertrophy, and $25(18 \%)$ had both. The main characteristics of participants with and without lipoatrophy or lipohypertrophy are compared on Table 2.

Lipoatrophy was seen more frequently in females $(17 / 28$, $60.7 \%)$ than in males $(39 / 108,36.1 \% ; P=0.03$, Table 2). Former and current smokers were more likely to have lipoatrophy. Other factors associated with lipoatrophy were duration of CART and treatment with stavudine and nonnucleosides. Participants with lipoatrophy had a longer known duration of HIV infection and a lower weight before initiation of CART compared to participants without lipoatrophy, but these differences were not statistically significant $(P=0.09$ and $P=0.064$, respectively, Table 2). There was no association between the Mediterranean diet score and lipoatrophy using bivariate analysis.

Of the 44 participants with lipohypertrophy $95 \%$ had fat deposition in the abdomen, and $84 \%$ reported fat deposition in the thorax. Of 28 females $19(67.9 \%)$ had signs of lipohypertrophy whereas of 108 males, 25 (23.2\%) had lipohypertrophy $(P<0.001$, Table 2$)$. Participants with moderate to high adherence to the Mediterranean diet (score $\geq 4$ ) had less frequent lipohypertrophy $(P=0.031$, Table 2). NNRTI use was more frequently associated with lipohypertrophy then PI use on bivariate analysis (Table 2). Of 45 patents who used PIs only 9 (20\%) had lipohypertrophy, while of 34 participants who used NNRTIs 16 (47\%) had lipohypertrophy. We also found that the following factors were associated with lipohypertrophy: longer known duration of HIV infection before CART was given, greater waist-to hip ratio, and a greater change in body mass index (Table 2).

Diet, energy intake and energy expenditure

Because of the gender difference in dietary intake and because of the low number of women in our study we report dietary intake only for 108 men. There was no statistically significant difference in consumption of the six 'beneficial' diet categories (vegetable, legumes, fruits and nuts, cereal, fish and monounsaturated fat to saturated fat ratio) between men with and without lipoatrophy or lipohypertrophy. However, participants without lipoatrophy consumed more fish than participants with lipoatrophy (median 38 g/day, IQR 25-45; vs. 28 g/day, IQR 17-45; $P=0.062$ ). For the two 'detrimental' categories (meat, which included poultry, and dairy) there was also no significant difference, though men with lipoatrophy consumed

Table 1 Number and severity of lipoatrophy and lipohypertrophy in 136 Croatian HIV-infected patients

\begin{tabular}{lccc}
\hline \multicolumn{2}{l}{ Severity and number of signs } & Lipoatrophy & Lipohypertrophy \\
\hline None & 1 & $48(35 \%)$ & $51(38 \%)$ \\
Only Subtle & 1 & 10 & 30 \\
& 1 & 14 & 1 \\
Moderate & $>1^{\mathrm{a}}$ & 8 & 10 \\
& 1 & 24 & 20 \\
Severe & $>1^{\mathrm{b}}$ & 3 & 3 \\
& & 29 & 21 \\
\hline Total & & 136 & 136 \\
\hline
\end{tabular}

The gray shading indicates patients included in the lipoatrophy group (56/136, 41\%) and in the lipohypertrophy group (44/136, 32\%). Twenty-five patients had both lipoatrophy and lipohypertrophy

${ }^{\text {a }}$ One moderate sign plus at least one subtle or one moderate

${ }^{b}$ One severe sign plus at least one subtle, one moderate or one severe sign 
Table 2 Main characteristics of 136 HIV-infected patients with and without lipoatrophy and lipohypertrophy [Values are percentages or medians (25th-75th percentile)]

\begin{tabular}{|c|c|c|c|c|c|c|}
\hline \multirow[t]{2}{*}{ Characteristics } & \multicolumn{3}{|l|}{ Lipoatrophy } & \multicolumn{3}{|c|}{ Lipohypertrophy } \\
\hline & Yes $(N=56)$ & No $(N=80)$ & $P$ & Yes $(N=44)$ & No $(N=92)$ & $P$ \\
\hline Male gender & 70 & 86 & 0.030 & 57 & 90 & $<.001$ \\
\hline Age at study inclusion (years) & $44(39-52)$ & $43(37-50)$ & 0.305 & $44(39-53)$ & $44(37-50)$ & 0.421 \\
\hline Former or current smoker & 77 & 58 & 0.042 & 71 & 64 & 0.562 \\
\hline \multicolumn{7}{|l|}{ Mode of infection } \\
\hline Heterosexual & 46 & 40 & 0.400 & 46 & 41 & 0.677 \\
\hline Male who has sex with men & 34 & 46 & & 39 & 42 & \\
\hline Injecting drug user & 9 & 10 & & 9 & 10 & \\
\hline Hemophiliac & 4 & 1 & & 5 & 1 & \\
\hline Unknown & 7 & 3 & & 2 & 5 & \\
\hline Mediterranean diet score $\geq 4$ & 62 & 71 & 0.352 & 55 & 74 & 0.031 \\
\hline \multicolumn{7}{|l|}{ Disease severity } \\
\hline $\begin{array}{l}\text { Known duration of HIV infection prior } \\
\text { to start of CART (months) }\end{array}$ & $9(1-53)$ & $3(0-33)$ & 0.090 & $29(1-65)$ & $3(0-26)$ & 0.011 \\
\hline History of AIDS defining illness & 52 & 44 & 0.386 & 50 & 46 & 0.714 \\
\hline Current HIV RNA count $<50$ copies/ml & 84 & 88 & 0.619 & 84 & 87 & 0.792 \\
\hline Peak HIV RNA $\geq 300,000$ copies $/ \mathrm{ml}$ & 59 & 53 & 0.488 & 52 & 57 & 0.714 \\
\hline Lowest ever CD4 count $\left(\right.$ cell $\left./ \mathrm{mm}^{3}\right) \leq 50$ & 48 & 40 & 0.382 & 43 & 43 & 1.000 \\
\hline \multicolumn{7}{|l|}{ Treatment } \\
\hline Duration of CART ( months) & $53(36-72)$ & $37(20-56)$ & 0.001 & $49(31-71)$ & $44(21-62)$ & 0.053 \\
\hline Protease inhibitor use $\geq 6$ months & 71 & 78 & 0.429 & 64 & 80 & 0.055 \\
\hline Non-nucleoside use $\geq 6$ months & 79 & 59 & 0.017 & 80 & 61 & 0.034 \\
\hline Stavudine use $\geq 6$ months & 73 & 46 & 0.003 & 61 & 55 & 0.580 \\
\hline \multicolumn{7}{|l|}{ Body measurements } \\
\hline Waist-to-hip ratio, males $>0.95$ or female $>0.8$ & 84 & 71 & 0.103 & 89 & 71 & 0.030 \\
\hline Change in BMI $>2 \mathrm{~kg} / \mathrm{m}^{2}$ & 61 & 54 & 0.483 & 71 & 50 & 0.028 \\
\hline Weight prior to CART (kg) & $63(54-72)$ & $68(60-78)$ & 0.064 & $62(54-75)$ & $66(57-74)$ & 0.243 \\
\hline
\end{tabular}

CART Combination antiretroviral therapy

more meat than men without lipoatrophy (134 g/day, IQR 100-153 vs. $114 \mathrm{~g} /$ day, IQR $89-140 ; P=0.08)$. Men without lipohypertrophy consumed more cereals than those with lipohypertrophy (282 g/day, IQR 246-325; vs. 256 g/ day, IQR 240-281; $P=0.021)$. There was no statistically significant difference in other food categories, however, men with no lipohypertrophy consumed more vegetables (364 g/day, IQR 260-447) and monounsaturated fat (36 g/ day, IQR 29-44) than men with lipohypertrophy (302 g/ day, IQR 247-374; $P=0.081$, and 32 g/day, IQR 26-39; $P=0.08$, respectively). Overall, 51 male participants (47.2\%) did not consume olive oil. Of 69 men without lipoatrophy, $48(70 \%)$ consumed olive oil, whereas of 39 men with lipoatrophy $20(51 \%)$ consumed olive oil $(P=0.066)$. Of 83 men without lipohypertrophy $58(70 \%)$ consumed olive oil, whereas of 25 men with lipohypertrophy $15(40 \%)$ consumed olive oil $(P=0.009)$. The median consumption of olive oil for men who used it was $17.7 \mathrm{~g} /$ day, IQR 13.5-24.8.
Alcohol consumption was similar in participants with and without lipoatrophy or lipohypertrophy, and there was also no difference in total energy intake and energy expenditure.

Multivariable analysis

Lipoatrophy was associated with stavudine use, gender, and duration of CART (Table 3). There was also a significant interaction between smoking and the Mediterranean diet score. Non-smokers who moderately or strongly adhered to the Mediterranean diet (score $\geq 4$ ) were less likely to have lipoatrophy then other subgroups (Table 3).

Lipohypertrophy was associated with a Mediterranean diet (score $<4$ ), gender, known duration of HIV infection prior to CART, and duration of CART (Table 3). Because the addition of PI or NNRTI use to the model gave a nonsignificant result we removed those variables from the final analysis. 
Table 3 Factors associated with lipoatrophy or lipohypertrophy among Croatian HIV infected patients treated with combination antiretroviral therapy (multivariable analysis)

\begin{tabular}{|c|c|c|c|}
\hline Body-shape feature and associated factors & OR & $95 \% \mathrm{CI}$ & $P$ \\
\hline \multicolumn{4}{|l|}{ Lipoatrophy } \\
\hline \multicolumn{4}{|l|}{ Stavudine use } \\
\hline Yes & 3.99 & $1.72-9.28$ & 0.001 \\
\hline No & 1.00 & & \\
\hline \multicolumn{4}{|l|}{ Gender } \\
\hline Male & 0.33 & $0.13-0.86$ & 0.023 \\
\hline Female & 1.00 & & \\
\hline Duration of CART (per 12 months) & 1.47 & $1.17-1.85$ & 0.001 \\
\hline \multicolumn{4}{|l|}{$\begin{array}{l}\text { Interaction of smoking }{ }^{\mathrm{a}} \text { and } \\
\text { Mediterranean diet score }\end{array}$} \\
\hline $\begin{array}{l}\text { Non-smoking in participants with } \\
\text { Mediterranean diet score } \geq 4\end{array}$ & 1.00 & & \\
\hline $\begin{array}{l}\text { Non-smoking in participants with } \\
\text { Mediterranean diet score }<4\end{array}$ & 4.53 & $0.86-23.92$ & 0.076 \\
\hline $\begin{array}{l}\text { Smoking in participants with } \\
\quad \text { Mediterranean diet score } \geq 4\end{array}$ & 3.42 & $1.21-9.67$ & 0.014 \\
\hline $\begin{array}{l}\text { Smoking in participants with } \\
\text { Mediterranean diet score }<4\end{array}$ & 4.39 & $1.35-14.26$ & 0.021 \\
\hline \multicolumn{4}{|l|}{ Lipohypertrophy } \\
\hline \multicolumn{4}{|l|}{ Mediterranean diet score } \\
\hline$<4$ & 1.00 & & \\
\hline$\geq 4$ & 0.30 & $0.12-0.75$ & 0.010 \\
\hline \multicolumn{4}{|l|}{ Gender } \\
\hline Male & 0.09 & $0.03-0.26$ & $<0.001$ \\
\hline Female & 1.00 & & \\
\hline $\begin{array}{l}\text { Known duration of HIV infection before } \\
\text { start of CART (per } 12 \text { months) }\end{array}$ & 1.17 & $1.05-1.31$ & 0.004 \\
\hline$<25$ months & 1.00 & & \\
\hline 25 months & 6.19 & $1.76-21.74$ & 0.005 \\
\hline
\end{tabular}

CART Combination antiretroviral therapy

${ }^{\text {a }}$ Current or former smoking

\section{Discussion}

We found that non-smokers who moderately or strongly adhered to a Mediterranean diet were least likely to have the clinical syndrome of lipoatrophy. Patients without the clinical syndrome of lipohypertrophy adhered to the Mediterranean diet more frequently than did participants with lipohypertrophy. Among individual components of the Mediterranean diet, only olive oil consumption showed a significant association with body fat change; those who consumed olive oil were less likely to have lipohypertrophy. These findings support the use of a combined Mediterranean diet score on the relationship between diet and body-shape changes, since individual components may each have only a small effect.
Except for the consumption of olive oil, which was very low in our study population, the median consumption of other food categories was similar to those reported from Greece by Trichopoulou et al. [16]. We did not use the polyunsaturated fat in the numerator of the lipid ratio as suggested by Trichopoulou et al. for populations with a low intake of olive oil [17] because the beneficial effects of polyunsaturated fat are still debated [27]. In our study, participants with moderate to high adherence to the Mediterranean diet consumed more legumes, fish, fruit and nuts, vegetables and had a higher ratio of monosaturated to saturated fat intake then participants with low adherence to the Mediterranean diet.

Data on diet and body-shape changes in HIV-infected patients are limited, and previous studies have not looked specifically at different food categories. An Australian study found no relationship between either saturated fat or total fat intake and body composition parameters characteristic of lipodystrophy in patients on CART [10]. Hadigan et al. reported that HIV-infected men, with or without fat redistribution, had similar dietary habits and did not differ from one another in total energy intake or macronutrient composition [11]. A nested case control study from the United States found that HIV patients on CART without fat deposition had greater overall energy intake, protein consumption, and total dietary fiber intake than patients with HIV-related fat deposition [14]. Roubenoff et al. described a patient in whom an exercise program and moderate-fat, low glicemic, high-fiber diet reversed central obesity [13]. We found that men without lipoatrophy tended to consume more fish and less meat then men with lipoatrophy. Men without lipohypertrophy consumed significantly more cereals then men with lipohypertrophy.

Only a few studies have looked at smoking as a risk factor for body-shape changes in HIV infected patients. In a cross-sectional study of body composition measured by dual energy x-ray absorptiometry (DEXA), Forrester and Gorbach found that Hispanic men who smoke had less total fat, less trunk fat, and more appendicular fat than nonsmokers [28]. Hendricks et al. reported that those without fat deposition tended not to be current smokers [14]. Smoking was associated with less visceral adipose tissue in women in the US-based Study of Fat Redistribution and Metabolic Change in HIV infection [9]. We found that lipoatrophy was more common in current and former smokers compared to nonsmokers. When this was adjusted for stavudine use, gender, and duration of CART, there was a significant interaction between smoking status and Mediterranean diet (Table 3) suggesting that diet and smoking habits combined may exert an effect on fat loss.

A number of risk factors for lipoatrophy have been identified in observational studies using multivariable analysis. They include, exposure to and duration of stavudine use [26, 
29-33], age [2, 4, 7, 9, 26, 29, 30, 32, 34, 35], presence of markers of severity of HIV disease [9, 26, 29, 30, 34-36], duration of therapy $[2,26,29,30,34]$, and white race $[9,26$, $34,36]$. We confirmed the role of stavudine and duration of CART as risk factors in our study. The role of stavudine and, to a lesser extent zidovudine, in lipoatrophy has been further established by findings from randomized controlled trials using lamivudine or emtricitabine with efavirenz and a thymidine analogue or tenofovir. Total limb fat was significantly less in patients treated with stavudine [37] and zidovudine [38] compared to tenofovir in a subgroup of patients with DEXA measurements. Several factors have been associated with lipohypertrophy: duration of therapy [26, 29, 30, 34], markers of disease severity [26, 30, 32], and age [26, 29, 30, 33]. Earlier studies found that PI use was associated with lipohypertrophy [30-32,34]. We did not find an association of PI use compared to NNRTI use in lipohypertrophy, a finding also reported in other more recent studies [7, 9, 35].

The principal limitation of our study was the cross-sectional design. Hence, we have not studied the change in adipose tissue over time. Confounding is inherent in any observational study especially when the sample size is relatively small. As the number of patients was relatively small in subgroups examining the association of the interaction of smoking and Mediterranean diet with lipoatrophy, we need additional studies to confirm these findings. We were able to adjust for the majority of known confounders for fat loss and fat gain, and we report results consistent with the majority of findings from larger studies. Also, the median duration of CART was long (47 months) which allowed us enough time to observe body fat changes. We included in our study $80 \%$ of all HIV infected Croatian patients who have been taking CART for at least 12 months. This in our view enables as to draw valid inferences for Croatians. Our findings, however, may not apply to other populations.

In summary, we found that 75 (55\%) of our patients who were taking CART for at least 12 months had clinical signs of body-shape changes. Croatian patients treated with CART and adhering to a Mediterranean diet might have a lower risk of developing body-shape changes. Our exploratory results need to be confirmed by larger studies and in other populations.

Open Access This article is distributed under the terms of the Creative Commons Attribution Noncommercial License which permits any noncommercial use, distribution, and reproduction in any medium, provided the original author(s) and source are credited.

\section{References}

1. Carr A, Samaras K, Burton S, et al. A syndrome of peripheral lipodystrophy, hyperlipidaemia and insulin resistance in patients receiving HIV protease inhibitors. AIDS. 1998;12:F51-8.
2. Thiebaut R, Daucourt V, Mercie P, et al. Lipodystrophy, metabolic disorders, and human immunodeficiency virus infection: Aquitaine Cohort, France, 1999. Groupe d'Epidemiologie Clinique du Syndrome d'Immunodeficience Acquise en Aquitaine. Clin Infect Dis. 2000;31:1482-7.

3. Chen D, Misra A, Garg A. Clinical review 153: lipodystrophy in human immunodeficiency virus-infected patients. J Clin Endocrinol Metab. 2002;87:4845-56.

4. Jacobson DL, Knox T, Spiegelman D, Skinner S, Gorbach S, Wanke C. Prevalence of, evolution of, and risk factors for fat atrophy and fat deposition in a cohort of HIV-infected men and women. Clin Infect Dis. 2005;40:1837-45.

5. Miller KD, Jones E, Yanovski JA, Shankar R, Feuerstein I, Falloon J. Visceral abdominal-fat accumulation associated with use of indinavir. Lancet. 1998;351:871-5.

6. Carr A, Miller J, Law M, Cooper DA. A syndrome of lipoatrophy, lactic acidaemia and liver dysfunction associated with HIV nucleoside analogue therapy: contribution to protease inhibitorrelated lipodystrophy syndrome. AIDS. 2000;14:F25-32.

7. Bacchetti P, Gripshover B, Grunfeld C, et al. Fat distribution in men with HIV infection. J Acquir Immune Defic Syndr. 2005;40:121-31.

8. Mulligan K, Parker RA, Komarow L, et al. Mixed patterns of changes in central and peripheral fat following initiation of antiretroviral therapy in a randomized trial. J Acquir Immune Defic Syndr. 2006;41:590-7.

9. Study of Fat Redistribution and Metabolic Change in HIV Infection (FRAM). Fat distribution in women with HIV infection. J Acquir Immune Defic Syndr 2006;42:562-71.

10. Batterham MJ, Garsia R, Greenop PA. Dietary intake, serum lipids, insulin resistance and body composition in the era of highly active antiretroviral therapy 'Diet FRS Study'. AIDS. 2000;14:1839-43.

11. Hadigan C, Jeste S, Anderson EJ, Tsay R, Cyr H, Grinspoon S. Modifiable dietary habits and their relation to metabolic abnormalities in men and women with human immunodeficiency virus infection and fat redistribution. Clin Infect Dis. 2001;33:710-7.

12. Barrios A, Blanco F, Garcia-Benayas T, et al. Effect of dietary intervention on highly active antiretroviral therapy-related dyslipemia. AIDS. 2002;16:2079-81.

13. Roubenoff R, Schmitz H, Bairos L, et al. Reduction of abdominal obesity in lipodystrophy associated with human immunodeficiency virus infection by means of diet and exercise: case report and proof of principle. Clin Infect Dis. 2002;34:390-3.

14. Hendricks KM, Dong KR, Tang AM, et al. High-fiber diet in HIV-positive men is associated with lower risk of developing fat deposition. Am J Clin Nutr. 2003;78:790-5.

15. Trichopoulou A. Traditional Mediterranean diet and longevity in the elderly: a review. Public Health Nutr. 2004;7:943-7.

16. Trichopoulou A, Costacou T, Bamia C, Trichopoulos D. Adherence to a Mediterranean diet and survival in a Greek population. N Engl J Med. 2003;348:2599-608.

17. Trichopoulou A, Orfanos P, Norat T, et al. Modified Mediterranean diet and survival: EPIC-elderly prospective cohort study. BMJ. 2005;330:991.

18. Lagiou P, Trichopoulos D, Sandin S, et al. Mediterranean dietary pattern and mortality among young women: a cohort study in Sweden. Br J Nutr. 2006;96:384-92.

19. Estruch R, Martinez-Gonzalez MA, Corella D, et al. Effects of a Mediterranean-style diet on cardiovascular risk factors: a randomized trial. Ann Intern Med. 2006;145:1-11.

20. Martinez-Gonzalez MA, Sanchez-Villegas A. The emerging role of Mediterranean diets in cardiovascular epidemiology: monounsaturated fats, olive oil, red wine or the whole pattern? Eur J Epidemiol. 2004;19:9-13. 
21. Trichopoulos D, Lagiou P. Mediterranean diet and cardiovascular epidemiology. Eur J Epidemiol. 2004;19:7-8.

22. Cottet V, Bonithon-Kopp C, Kronborg O, et al. Dietary patterns and the risk of colorectal adenoma recurrence in a European intervention trial. Eur J Cancer Prev. 2005;14:21-9.

23. La Vecchia C. Mediterranean diet and cancer. Public Health Nutr. 2004;7:965-8.

24. Craig CL, Marshall AL, Sjostrom M, et al. International physical activity questionnaire: 12-country reliability and validity. Med Sci Sports Exerc. 2003;35:1381-95.

25. Ainsworth BE, Haskell WL, Whitt MC, et al. Compendium of physical activities: an update of activity codes and MET intensities. Med Sci Sports Exerc. 2000;32:S498-504.

26. Lichtenstein KA, Ward DJ, Moorman AC, et al. Clinical assessment of HIV-associated lipodystrophy in an ambulatory population. AIDS. 2001;15:1389-98.

27. Hooper L, Thompson RL, Harrison RA, et al. Risks and benefits of omega 3 fats for mortality, cardiovascular disease, and cancer: systematic review. BMJ. 2006;332:752-60.

28. Forrester JE, Gorbach SL. Fat distribution in relation to drug use, human immunodeficiency virus (HIV) status, and the use of antiretroviral therapies in Hispanic patients with HIV infection. Clin Infect Dis. 2003;37(Suppl 2):S62-8.

29. Martinez E, Mocroft A, Garcia-Viejo MA, et al. Risk of lipodystrophy in HIV-1-infected patients treated with protease inhibitors: a prospective cohort study. Lancet. 2001;357:592-8.

30. Miller J, Carr A, Emery S, et al. HIV lipodystrophy: prevalence, severity and correlates of risk in Australia. HIV Med. 2003;4:293-301.
31. Heath KV, Hogg RS, Singer J, Chan KJ, O'Shaughnessy MV, Montaner JS. Antiretroviral treatment patterns and incident HIVassociated morphologic and lipid abnormalities in a populationbased chort. J Acquir Immune Defic Syndr. 2002;30:440-7.

32. Saves M, Raffi F, Capeau J, et al. Factors related to lipodystrophy and metabolic alterations in patients with human immunodeficiency virus infection receiving highly active antiretroviral therapy. Clin Infect Dis. 2002;34:1396-405.

33. Joly V, Flandre P, Meiffredy V, et al. Increased risk of lipoatrophy under stavudine in HIV-1-infected patients: results of a substudy from a comparative trial. AIDS. 2002;16:2447-54.

34. Bogner JR, Vielhauer V, Beckmann RA, et al. Stavudine versus zidovudine and the development of lipodystrophy. J Acquir Immune Defic Syndr. 2001;27:237-44.

35. Young J, Rickenbach M, Weber R, et al. Body fat changes among antiretroviral-naive patients on PI- and NNRTI-based HAART in the Swiss HIV cohort study. Antivir Ther. 2005;10:73-81.

36. Lichtenstein KA, Delaney KM, Armon C, et al. Incidence of and risk factors for lipoatrophy (abnormal fat loss) in ambulatory HIV-1-infected patients. J Acquir Immune Defic Syndr. 2003;32:48-56.

37. Gallant JE, Staszewski S, Pozniak AL, et al. Efficacy and safety of tenofovir DF vs stavudine in combination therapy in antiretroviralnaive patients: a 3-year randomized trial. JAMA. 2004;292:191201.

38. Gallant JE, DeJesus E, Arribas JR, et al. Tenofovir DF, emtricitabine, and efavirenz vs. zidovudine, lamivudine, and efavirenz for HIV. N Engl J Med. 2006;354:251-60. 